\title{
Perturbed Iterative Algorithms for Split General Mixed Variational Inequality Problem
}

\author{
Yali Zhao, Qian Zhang and Shuyi Zhang \\ College of Mathematics and Physics, Bohai University, Jinzhon, Liaoning 121013, China
}

\begin{abstract}
In this paper, we introduce a split general mixed variational inequality problem, which is a natural extension of a split variational inequality problem, split general quasi-variational inequality problem in Hilbert spaces. Using the resolvent operator technique, we propose two classes of perturbed iterative algorithms for the split general mixed variational inequality problem. Further, we discuss the convergence criteria of the iterative algorithms. The results presented here extend and improve many previously known results in this area.
\end{abstract}

Keyword-split general mixed variational inequality problem; split general quasi-variational inequality problem; perturbed iterative algorithm;convergence

\section{INTRODUCTION}

In a recent paper [1], Kazmi has developed an iterative algorithm for finding approximate solution for a new split general quasi-variational inequality problem in Hilbert spaces. The aim of this work is to extend his idea to more general problem. Throughout the paper unless stated otherwise, for each $i \in\{1,2\}$, let $H_{i}$ be a real Hilbert space with inner product $\langle\cdot$, and norm $\|\cdot\|$, let $f_{i}: H_{i} \rightarrow H_{i}, g_{i}: H_{i} \rightarrow H_{i}$ be continuous mappings with $\operatorname{Im} g_{i} \cap \operatorname{dom} \varphi_{i} \neq \phi$, Let $A: H_{1} \rightarrow H_{2}$ be a bounded linear operator with its adjoint operator $A^{*}$. We consider the following problem: Find $x_{1}{ }^{*} \in H_{1}$ such that $g_{1}\left(x_{1}{ }^{*}\right) \in \operatorname{dom} \varphi_{1}$ and

$$
\left\langle f_{1}\left(x_{1}^{*}\right), x_{1}-g_{1}\left(x_{1}^{*}\right)\right\rangle \geq \varphi_{1}\left(g_{1}\left(x_{1}^{*}\right)\right)-\varphi_{1}\left(x_{1}\right), \quad \forall x_{1} \in H_{1},
$$

and such that $x_{2}^{*}=A x_{1}^{*} \in H_{2}, g_{2}\left(x_{2}^{*}\right) \in \operatorname{dom} \varphi_{2}$ solves

$$
\left\langle f_{2}\left(x_{2}^{*}\right), x_{2}-g_{2}\left(x_{2}^{*}\right)\right\rangle \geq \varphi_{2}\left(g_{2}\left(x_{2}^{*}\right)\right)-\varphi_{2}\left(x_{2}\right), x_{2} \in H_{2} \text {. }
$$

We call problem (1)-(2)the split general mixed variational inequality problem (in short, SpGMVIP). SpGMVIP(1)-(2) amounts to saying: find a solution of general mixed variational inequality problem (1) image under a given bounded linear operator is a solution of general mixed variational inequality problem (2). For convenience, we denote the solution set of SpGMVIP(1)-(2) by

$$
\Gamma=\left\{x_{1}^{*} \in H_{1} \mid x_{1}^{*} \text { solves(1) and } A x_{1}^{*} \in H_{2} \text { solves (2) }\right\}
$$

Next, we give some special cases of SpGMVIP (1)-(2).
If we set $g_{i}=I_{i}$, where $I_{i}$ is an identity operator on $H_{i}$, then SpGMVIP(1)-(2) is reduced to the following split mixed variational inequality problem (In short, SpMVIP): Find $x_{1}^{*} \in H_{1}$ such that

$$
\left\langle f_{1}\left(x_{1}^{*}\right), x_{1}-x_{1}^{*}\right\rangle \geq \varphi_{1}\left(x_{1}^{*}\right)-\varphi_{1}\left(x_{1}\right), \forall x_{1} \in H_{1},
$$

and such that $x_{2}^{*}=A x_{1}^{*} \in H_{2}$ solves

$$
\left\langle f_{2}\left(x_{2}^{*}\right), x_{2}-x_{2}^{*}\right\rangle \geq \varphi_{2}\left(x_{2}^{*}\right)-\varphi_{2}\left(x_{2}\right), \forall x_{2} \in H_{2},
$$

which appears to be new one.

$$
\text { If we set } \varphi_{i}(\cdot)=\delta_{C_{i}}\left(\cdot-m_{i}\left(x_{i}^{*}\right)\right)=\delta_{C_{i}+m_{i}\left(x_{i}^{*}\right)}(\cdot), m_{i}: H_{i} \rightarrow H_{i} \text { is a }
$$
single-valued mapping, where $C_{i}\left(x_{i}^{*}\right)=C_{i}+m_{i}\left(x_{i}^{*}\right)$, and $C_{i}$ is a closed convex subset of $H_{i}$, then SpMVIP (1)-(2) is reduced to the following split general quasi-variational inequality problem (in short, SpGQVIP): Find $x_{1}^{*} \in H_{1}$, such that $g_{1}\left(x_{1}^{*}\right) \in C_{i}\left(x_{1}^{*}\right)$ and

$$
\left\langle f_{1}\left(x_{1}^{*}\right), x_{1}-g_{1}\left(x_{1}^{*}\right)\right\rangle \geq 0, \forall x_{1} \in C_{1}\left(x_{1}^{*}\right),
$$

and such that $x_{2}^{*}=A x_{1}^{*}, g_{2}\left(x_{2}^{*}\right) \in C_{2}\left(x_{2}^{*}\right)$ solves

$$
\left\langle f_{2}\left(x_{2}^{*}\right), x_{2}-g_{2}\left(x_{2}^{*}\right)\right\rangle \geq 0, \forall x_{2} \in C_{2}\left(x_{2}^{*}\right) \text {. }
$$

This problem was introduced and studied by Kazmi in [1] and he exhibited split quasi-variational inequality problem, split general variational inequality problem and quasi-variaeional inequality problem as special cases of SpGQVIP (5)-(6). For details, see reference[1].

$$
\text { If } \varphi_{i}=\delta_{C_{i}} \text { the indicator function of a closed convex set }
$$
$C_{i} \subset H_{i}, g_{i}=I_{i}$ the identity mapping $H_{i}$, then SpGMVIP (1)-(2) is reduced to the following split general variational inequality problem (in short, SpVIP): Find $x_{1}^{*} \in H_{1}$, such that

$$
\left\langle f_{1}\left(x_{1}^{*}\right), x_{1}-x_{1}^{*}\right\rangle \geq 0, \forall x_{1} \in C_{1}
$$

and $x_{2}^{*}=A x_{1}^{*} \in H_{2}$ solves

$$
\left\langle f_{2}\left(x_{2}^{*}\right), x_{2}-x_{2}^{*}\right\rangle \geq 0, \forall x_{2} \in C_{2},
$$


which has been introduced and studied by Censor, Gibali and Reich[2]. It is worth noting that SpGMVIP(1)-(2) is quite general and includes as special cases split minimization between two spaces so that the image of a minimize of a given function, under a bounded linear operator, is a minimizer of a given function, under a bounded linear operator, is a minimizer of another function, split zero problem and the split feasibility problem which have already been studied and used in practice as a model in the intensity-moducated radiation therapy planning, see[ $3,4,5]$.

In a word, SpGMVIP is more general, which is one of our motivations to write this paper. By using the resolvent operator technique about the maximal monotone mapping, we propose two classes of perturbed iterative algorithms taking into account a possible in exact computation for SpGMVIP (1)-(2) and discuss the convergence criteria of these iterative algorithms. The results presented here extend and improve the previously known results in this area.

\section{Perturbed Iterative Algorithms}

To begin with, let us transform SpGMVIP (1)-(2) into fixed point problems.

Lemma 2.1. $x_{1}^{*} \in \Gamma$ if and only if ${ }^{*}$ satisfies the following relations

$$
\begin{aligned}
& g_{1}\left(x_{1}^{*}\right)=J_{\rho_{1}}^{\partial \rho_{1}}\left(g_{1}\left(x_{1}^{*}\right)-\rho_{1} f_{1}\left(x_{1}^{*}\right)\right), \\
& g_{2}\left(A x_{1}^{*}\right)=J_{\rho_{2}}^{\partial \varphi_{2}}\left(g_{2}\left(A x_{1}^{*}\right)-\rho_{2} f_{2}\left(A x_{1}^{*}\right)\right),
\end{aligned}
$$

where $\rho_{i}>0$ is a constant and $J_{\rho_{i}}^{\partial \varphi_{i}}:=\left(I+\rho_{i} \partial \varphi_{i}\right)^{-1}$ is the

resolvent operator of the maximal monotone mapping $\partial \varphi_{i}$,

noting that ${ }^{\partial \varphi_{i}}$ denotes the subdifferential of a proper, convex and lower semi-continuous function $\varphi_{i}: H \rightarrow R \bigcup\{+\infty\}$.

Proof From definition of $J_{\rho_{i}}^{\partial \varphi_{i}}$, It follows from (9)that

$$
g_{1}\left(x_{1}^{*}\right)-\rho_{1} f_{1}\left(x_{1}^{*}\right) \in g_{1}\left(x_{1}^{*}\right)+\rho_{1} \partial \varphi_{1}\left(g_{1}\left(x_{1}^{*}\right)\right),
$$

then $-f_{1}\left(x_{1}^{*}\right) \in \partial \varphi_{1}\left(g_{1}\left(x_{1}^{*}\right)\right)$, definition of $\partial \varphi_{\text {implies }}$

$$
\varphi_{1}\left(x_{1}\right) \geq \varphi_{1}\left(g_{1}\left(x_{1}^{*}\right)\right)+\left\langle-f_{1}\left(x_{1}^{*}\right), x_{1}-g_{1}\left(x_{1}^{*}\right)\right\rangle, \forall x_{1} \in H_{1} .
$$

this is,

$$
\left\langle f_{1}\left(x_{1}^{*}\right), x_{1}-g_{1}\left(x_{1}^{*}\right)\right\rangle \geq \varphi_{1}\left(g_{1}\left(x_{1}^{*}\right)\right)-\varphi_{1}\left(x_{1}\right), \forall x_{1} \in H_{1} .
$$

thus $x_{1}^{*}$ is a solution of (1). Similarly, it is easy to know $A x_{1}^{*}$ solves(2), hence ${ }^{x_{1}^{*}} \in \Gamma$. The converse relation is obvious, so is omitted, completing the proof.

Based on Lemma 2.1, we can propose the following perturbed iterative algorithms for approximating a solution to
SpGMVIP(1)-(2). Let $\left\{\alpha^{n}\right\} \subseteq(0,1)$ be a sequence such that $\sum_{n=1}^{\infty} \alpha^{n}=\infty$ and let $\rho_{1}, \rho_{2}, \gamma$ be the parameters with positive values.

Algorithm 2.1. Given $x_{1}^{0} \in H_{1}$, compute the iterative sequence $\left\{x_{1}^{n}\right\}$ defined by the iterative schemes

$$
\begin{gathered}
g_{1}\left(y^{n}\right)=J_{\rho_{1}}^{\partial \varphi_{1}^{n}}\left(g_{1}\left(x_{1}^{n}\right)-\rho_{1} f_{1}\left(x_{1}^{n}\right)\right), \\
g_{2}\left(z^{n}\right)=J_{\rho_{2}}^{\partial \rho_{2}^{n}}\left(g_{2}\left(A y^{n}\right)-\rho_{2} f_{2}\left(A y^{n}\right)\right), \\
x_{1}^{n+1}=\left(1-\alpha^{n}\right) x_{1}^{n}+\alpha^{n}\left[y^{n}+\gamma A^{*}\left(z^{n}-A y^{n}\right)\right]+\alpha^{n} e^{n},
\end{gathered}
$$

for all $n=0,1,2, \cdots, \rho_{1}, \rho_{2}, \gamma>0$ and take into account a possible inexact computation, an $\left\|e^{n}\right\| \rightarrow 0(n \rightarrow \infty)$. error $e^{n}$ is added in the right hand side of(13) withMoreover, we consider other perturbations by replacing in (11) and (12) $\varphi_{i}$ by $\varphi_{i}^{n}$, where the sequence $\left\{\varphi_{i}^{n}\right\}$ approximates $\varphi_{i},\left\{\varphi_{i}^{n}\right\}$ is a collection of proper convex semi-continuous functions on ${ }^{H_{i}}$.

If $\varphi_{i}(\cdot)=\delta_{C_{i}\left(x_{i}^{*}\right)}(\cdot)$, where $C_{i}\left(x_{i}^{*}\right)$ is same as the above, $e^{n}=0$, then Algorithm 2.1 is reduced to the following algorithm for SpGQVIP:

Algorithm 2.2. Given $x_{1}^{0} \in H_{1}$, compute the iterative sequence $\left\{x_{1}^{n}\right\}$ defined by the iterative schemes

$$
\begin{gathered}
g_{1}\left(y^{n}\right)=P_{C_{1}\left(x_{1}^{n}\right)}\left(g_{1}\left(x_{1}^{n}\right)-\rho_{1} f_{1}\left(x_{1}^{n}\right)\right), \\
g_{2}\left(z^{n}\right)=P_{C_{2}\left(A^{n}\right)}\left(g_{2}\left(A y^{n}\right)-\rho_{2} f_{2}\left(A y^{n}\right)\right), \\
x_{1}^{n+1}=\left(1-\alpha^{n}\right) x_{1}^{n}+\alpha^{n}\left[y^{n}+\gamma A^{*}\left(z^{n}-A y^{n}\right)\right],
\end{gathered}
$$

for all $n=0,1,2, \cdots, \rho_{1}, \rho_{2}, \gamma>0$, where $P_{c_{i}}$ is the metric projection of $H_{i}$ on to ${ }^{C_{i}}$, and it is well known that $P_{C_{i}}$ is a nonexpansive mapping. Algorithm 2.2 was proposed by Kazmi [1] for SpGQVIP.

Observe that (9)and (10) can change into the following :

$$
x_{i}^{*}=x_{i}^{*}-g_{i}\left(x_{i}^{*}\right)+J_{\rho_{i}}^{\partial \varphi_{i}}\left(g_{i}\left(x_{i}^{*}\right)-\rho_{i} f_{i}\left(x_{i}^{*}\right)\right), i=1,2,
$$

where $x_{2}^{*}=A x_{1}^{*}, \rho_{i}>0$ is a constant. In view of the above equations, we can propose another perturbed iterative algorithm for SpGMVIP.

Algorithm 2.3. Given $x_{1}^{0} \in H$, compute the iterative sequence $\left\{x_{1}^{n}\right\}$ defined by the iterative schemes

$$
\begin{aligned}
& y^{n}=x_{1}^{n}-g_{1}\left(x_{1}^{n}\right)+J_{\rho_{1}}^{\partial \varphi_{1}^{n}}\left(g_{1}\left(x_{1}^{n}\right)-\rho_{1} f_{1}\left(x_{1}^{n}\right)\right), \\
& z^{n}=A y^{n}-g_{2}\left(y^{n}\right)+J_{\rho_{2}}^{\partial \rho_{2}^{n}}\left(g_{2}\left(A y^{n}\right)-\rho_{2} f_{2}\left(A y^{n}\right)\right),
\end{aligned}
$$




$$
x_{1}^{n+1}=\left(1-\alpha^{n}\right) x_{1}^{n}+\alpha^{n}\left[y^{n}+\gamma A^{*}\left(z^{n}-A y^{n}\right)\right]+\alpha^{n} e^{n},
$$

for all $n=0,1,2, \cdots, \rho_{1}, \rho_{2}, \gamma>0, e^{n}$ is an error term and $\left\|e^{n}\right\| \rightarrow 0(n \rightarrow \infty)$.

In order to obtain our main results, we need the following definition, Assumption and lemmas.

Definition 2.1. A nonlinear mapping $f: H_{1} \rightarrow H_{1}$ is said to be

(i) $\alpha$-strongly monotone if there exists a constant $\alpha>0$ such that

$$
\langle f(x)-f(y), x-y\rangle \geq \alpha\|x-y\|^{2}, \forall x, y \in H_{1} .
$$

(ii) $\beta$-Lipschitz continuous if there exists a constant $\beta>0$ such that

$$
\|f(x)-f(y)\| \leq \beta\|x-y\|, \forall x, y \in H_{1} .
$$

Remark 2.1. It is easy to know that if $f: H_{1} \rightarrow H$ is $\alpha$ -strongly monotone and $\beta$-Lipschitz continuous then $\alpha \leq \beta$. Assumption 2.2. For $i \in\{1,2\}$, let $\varphi_{i}: H_{i} \rightarrow \bar{R}=R \cup\{+\infty\}$ be a proper, convex and lower semi-continuous function, $\left\{\varphi_{i}^{n}\right\}$ approximate $\varphi_{i}$ and satisfies the condition:

$$
\lim _{n \rightarrow \infty}\left\|J_{\rho_{i}}^{\partial \rho_{i}^{n}}\left(v_{i}\right)-J_{\rho_{i}}^{\partial \varphi_{i}}\left(v_{i}\right)\right\|=0, \forall v_{i} \in H_{i}
$$

Lemma 2.3([6]). Let ${ }^{\left\{a_{k}\right\}}$ be a sequence of nonnegative real numbers satisfying the condition

$$
a_{k+1} \leq\left(1-m_{k}\right) a_{k}+m_{k} \delta_{k}, \forall k \geq 0 .
$$

where $\left\{m_{k}\right\},\left\{\delta_{k}\right\}$ are sequences of real numbers such that

(i) $\left\{m_{k}\right\} \subset[0,1]$ and $\sum_{k=0}^{\infty} m_{k}=\infty$,or, equivalently,

$$
\prod_{k=0}^{\infty}\left(1-m_{k}\right):=\lim _{k \rightarrow \infty} \prod_{j=0}^{k}\left(1-m_{i}\right)=0
$$

(ii) $\limsup _{k \rightarrow \infty} \delta_{k} \leq 0$, or (ii)' $\sum_{k=0}^{\infty} \delta_{k} m_{k}$ is convergent.

Then $\lim _{k \rightarrow \infty} a_{k}=0$.

Lemma 2.4. Let $H$ be a real Hilbert space, for all $x, y \in H$, the following hold:

$$
\begin{gathered}
\|x+y\|^{2} \leq\|x\|^{2}+2\langle y, x+y\rangle, \\
\|x+y\|^{2}=\|x\|^{2}+2\langle x, y\rangle+\|y\|^{2} .
\end{gathered}
$$

\section{MAIN RESUlTS}

Theorem 3.1. For each $i \in\{1,2\}$, let $g_{i}: H_{i} \rightarrow H_{i}$ be $\delta_{i}$-Lipschitz continuous such that $\left(g_{i}-I_{i}\right)$ is $\delta_{i}$-strongly monotone, where $I_{i}$ is the identity operator on $H_{i}$. Let $f_{i}: H_{i} \rightarrow H_{i}$ be $\alpha_{i}$ -strongly monotone with respect to $g_{i}$ and $\beta_{i}$-Lipschitz continuous. Let $A: H_{1} \rightarrow H_{2}$ be a bounded linear operator and let $A^{*}$ be its adjoint operator. Suppose $x_{1}^{*} \in \Gamma$ and Assumption 2.2 holds. Then the sequence $\left\{x_{1}^{n}\right\}$ generated by Algorithm 2.1 converges strongly to $x_{1}^{*}$ provided that the constant $\rho_{i}$ and $\gamma$ satisfy the conditions

$$
\begin{aligned}
& \left|\rho_{1}-\frac{\alpha}{\beta_{1}^{2}}\right|<\frac{\sqrt{\tau_{1}^{2}+\alpha_{1}^{2}-\delta_{1}^{2}}}{\beta_{1}^{2}}, \quad \delta_{1}<\sqrt{\tau_{1}^{2}+\alpha_{1}^{2}}, \\
& \gamma\|A\|^{2} \theta_{1} \theta_{2}<1-\theta_{1}, \gamma \in\left(0, \frac{2}{\|A\|^{2}}\right), \\
& \theta_{i}=\tau_{i} \sqrt{\delta_{i}^{2}-2 \rho_{i} \alpha_{i}+\rho_{i}^{2} \beta_{i}^{2}}, \tau_{i}=\frac{1}{\sqrt{1+2 \sigma_{i}}}, \rho_{i}>0, i=1,2 .
\end{aligned}
$$

Proof Since $x_{1}^{*} \in \Gamma$, then $x_{i}^{*} \in H_{i}$ such that

$g_{i}\left(x_{i}^{*}\right) \in \operatorname{dom} \varphi_{i}\left(g_{i}\left(x_{i}\right)\right)$ and

$$
\begin{gathered}
g_{1}\left(x_{1}^{*}\right)=J_{\rho_{1}}^{\partial \rho_{1}}\left(g_{1}\left(x_{1}^{*}\right)-\rho_{1} f_{1}\left(x_{1}^{*}\right)\right), \\
g_{2}\left(A x_{1}^{*}\right)=J_{\rho_{2}}^{\partial \varphi_{2}}\left(g_{2}\left(A x_{1}^{*}\right)-\rho_{2} f_{2}\left(A x_{1}^{*}\right)\right),
\end{gathered}
$$

for $\rho_{i}>0$ and $x_{2}^{*}=A x_{1}^{*}$. From Algorithm 2.1(11), Assumption 2.2 and (20), we have

$$
\begin{aligned}
& \left\|g_{1}\left(y^{n}\right)-g_{1}\left(x_{1}^{*}\right)\right\| \\
& =\left\|J_{\rho_{1}}^{\partial \rho_{1}^{n}}\left(g_{1}\left(x_{1}^{n}\right)-\rho_{1} f_{1}\left(x_{1}^{n}\right)\right)-J_{\rho_{1}}^{\partial \rho_{1}}\left(g_{1}\left(x_{1}^{*}\right)-\rho_{1} f_{1}\left(x_{1}^{*}\right)\right)\right\| \\
& \leq\left\|J_{\rho_{1}}^{\partial \rho_{1}^{n}}\left(g_{1}\left(x_{1}^{n}\right)-\rho_{1} f_{1}\left(x_{1}^{n}\right)\right)-J_{\rho_{1}}^{\partial \rho_{1}^{n}}\left(g_{1}\left(x_{1}^{*}\right)-\rho_{1} f_{1}\left(x_{1}^{*}\right)\right)\right\| \\
& +\left\|J_{\rho_{1}}^{\partial \rho \rho_{1}}\left(g_{1}\left(x_{1}^{*}\right)-\rho_{1} f_{1}\left(x_{1}^{*}\right)\right)-J_{\rho_{1}}^{\partial \rho_{1}}\left(g_{1}\left(x_{1}^{*}\right)-\rho_{1} f_{1}\left(x_{1}^{*}\right)\right)\right\| \\
& \leq\left\|g_{1}\left(x_{1}^{n}\right)-g_{1}\left(x_{1}^{*}\right)-\rho_{1}\left(f_{1}\left(x_{1}^{n}\right)-f_{1}\left(x_{1}^{*}\right)\right)\right\|+\varepsilon_{1}^{n},
\end{aligned}
$$

where $\quad \varepsilon_{1}^{n}=\left\|J_{\rho_{1}}^{\partial \rho_{1}^{n}}\left(g_{1}\left(x_{1}^{*}\right)-\rho_{1} f_{1}\left(x_{1}^{*}\right)\right)-J_{\rho_{1}}^{\partial \rho_{1}}\left(g_{1}\left(x_{1}^{*}\right)-\rho_{1} f_{1}\left(x_{1}^{*}\right)\right)\right\| \quad$ and $\lim _{n \rightarrow \infty} \varepsilon_{1}^{n}=0$ owns to Assumption 2.2. Now, using the facts that $f_{1}$ is $\alpha_{1}$-strongly monotone with respect to $g_{1}$ and

$\beta_{i}$-Lipschitz continuous, and $g_{1}$ is $\delta_{i}$-Lipschitz continuous,we have

$$
\begin{aligned}
& \left\|g_{1}\left(x_{1}^{n}\right)-g_{1}\left(x_{1}^{*}\right)-\rho_{1}\left(f_{1}\left(x_{1}^{n}\right)-f_{1}\left(x_{1}^{*}\right)\right)\right\|^{2}=\left\|g_{1}\left(x_{1}^{n}\right)-g_{1}\left(x_{1}^{*}\right)\right\|^{2} \\
& -2 \rho_{1}\left\langle f_{1}\left(x_{1}^{n}\right)-f_{1}\left(x_{1}^{*}\right), g_{1}\left(x_{1}^{n}\right)-g_{1}\left(x_{1}^{*}\right)\right\rangle+\rho_{1}^{2}\left\|f_{1}\left(x_{1}^{n}\right)-f_{1}\left(x_{1}^{*}\right)\right\|^{2} \\
& \leq\left(\delta_{1}^{2}-2 \rho_{1} \alpha_{1}+\rho_{1}^{2} \beta_{1}^{2}\right)\left\|x_{1}^{n}-x_{1}^{*}\right\|^{2} .
\end{aligned}
$$

Combing (22)and(23), we have 


$$
\left\|g_{1}\left(y^{n}\right)-g_{1}\left(x_{1}^{*}\right)\right\| \leq \sqrt{\delta_{1}^{2}-2 \rho_{1} \alpha_{1}+\rho_{1}^{2} \beta_{1}^{2}}\left\|x_{1}^{n}-x_{1}^{*}\right\|+\varepsilon_{1}^{n} .
$$

Since $\left(g_{1}-I_{i}\right)$ is $\sigma_{1}$-strongly monotone, we have

$$
\begin{aligned}
\left\|y^{n}-x_{1}^{*}\right\|^{2} & \leq\left\|g_{1}\left(y^{n}\right)-g_{1}\left(x_{1}^{*}\right)\right\|^{2}-2\left\langle\left(g_{1}-I_{1}\right) y^{n}-\left(g_{1}-I_{1}\right) x_{1}^{*}, y^{n}-x_{1}^{*}\right\rangle \\
& \leq\left\|g_{1}\left(y^{n}\right)-g_{1}\left(x_{1}^{*}\right)\right\|^{2}-2 \sigma_{1}\left\|y^{n}-x_{1}^{*}\right\|^{2},
\end{aligned}
$$

which implies

$$
\left\|y^{n}-x_{1}^{*}\right\| \leq \tau_{1}\left\|g_{1}\left(y^{n}\right)-g_{1}\left(x_{1}^{*}\right)\right\|,
$$

where $_{\tau_{1}}=\frac{1}{\sqrt{1+2 \sigma_{1}}}$. From(24)and(25), we get

$$
\left\|y^{n}-x_{1}^{*}\right\| \leq \theta_{1}\left\|x_{1}^{n}-x_{1}^{*}\right\|+\tau_{1} \varepsilon_{1}^{n},
$$

where $\theta_{1}=\tau_{1} \sqrt{\delta_{1}^{2}-2 \rho_{1} \alpha_{1}+\rho_{1}^{2} \beta_{1}^{2}}$. Similarly, from Algorithm 2.1(12), Assumption 2.2 and (21) and using the facts that $f_{2}$ is $\alpha_{2}$-strongly monotone with respect to $g_{2}$

and $\beta_{2}$-Lipschitz continuous, $\left(g_{2}-I_{2}\right)$ is $\sigma_{2}$-strongly monotone, and $g_{2}$ is $\delta_{2}$-Lipschitz continuous, we have

$$
\left\|g_{2}\left(z^{n}\right)-g_{2}\left(A x_{1}^{*}\right)\right\| \leq \sqrt{\delta_{2}^{2}-2 \rho_{2} \alpha_{2}+\rho_{2}^{2} \beta_{2}^{2}}\left\|A y^{n}-A x_{1}^{*}\right\|,
$$

and

$$
\left\|z^{n}-A x_{1}^{*}\right\| \leq \theta_{2}\left\|A y^{n}-A x_{1}^{*}\right\|+\tau_{2} \varepsilon_{2},
$$

where

$$
\begin{gathered}
\tau_{2}=\frac{1}{\sqrt{2 \sigma_{2}+1}}, \theta_{2}=\tau_{2} \sqrt{\delta_{2}^{2}-2 \rho_{2} \alpha_{2}+\rho_{2}^{2} \beta_{2}^{2}}, \\
\varepsilon_{2}^{n}=\left\|J_{\rho_{2} \rho_{2}^{n}}\left(g_{2}\left(A x_{1}^{*}\right)-\rho_{2} f_{2}\left(A x_{1}^{*}\right)\right)-J_{\rho_{2}}^{\partial \rho_{2}}\left(g_{2}\left(A x_{1}^{*}\right)-\rho_{2} f_{2}\left(A x_{1}^{*}\right)\right)\right\|,
\end{gathered}
$$

and $\lim _{n \rightarrow \infty} \varepsilon_{2}^{n}=0$ owns to Assumption 2.2. From Algorithm 2.1(13), we obtain

$$
\begin{aligned}
& \left\|x_{1}^{n+1}-x_{1}^{*}\right\| \\
& \leq\left(1-\alpha^{n}\right)\left\|x_{1}^{n}-x_{1}^{*}\right\|+\alpha^{n} \|\left[y^{n}-x_{1}^{*}-\gamma A^{*}\left(A y^{n}-A x_{1}^{*}\right)\|+\gamma\| A^{*}\left(z^{n}-A x_{1}^{*}\right)\left\|+\alpha^{n} \mid e^{n}\right\| .\right.
\end{aligned}
$$

Further, using the definition of $A^{*}$, the face that $A^{*}$ is a bounded linear operator with $\left\|A^{*}\right\|=\|A\|$, and the given condition on $\gamma$, we have

$$
\begin{aligned}
& \left\|y^{n}-x_{1}^{*}-\lambda A^{*}\left(A y^{n}-A x_{1}^{*}\right)\right\|^{2} \\
= & \left\|y^{n}-x_{1}^{*}\right\|^{2}-2 \gamma\left(y^{n}-x_{1}^{*}, A^{*}\left(A y^{n}-A x_{1}^{*}\right)\right\rangle+\gamma^{2}\left\|A^{*}\left(A y^{n}-A x_{1}^{*}\right)\right\|^{2} \\
\leq & \left\|y^{n}-x_{1}^{*}\right\|^{2}-\gamma\left(2-\gamma\|A\|^{2}\right)\left\|A y^{n}-A x_{1}\right\|^{2} \leq\left\|y^{n}-x_{1}^{*}\right\|^{2},
\end{aligned}
$$

and, using(3.9), we have

$$
\begin{aligned}
\left\|A^{*}\left(z^{n}-A x_{1}^{*}\right)\right\| & \leq\|A\|\left\|z^{n}-A x_{1}^{*}\right\| \leq \theta_{2}\|A\| A y^{n}-A x_{1}^{*}\|+\| A \| \tau_{2} \varepsilon_{2}^{n} \\
& \leq \theta_{2}\|A\|^{2}\left\|y^{n}-x_{1}^{*}\right\|+\|A\| \tau_{2} \varepsilon_{2}^{n} .
\end{aligned}
$$

It follows from (29)-(31), we obtain

$$
\begin{aligned}
& \left\|x_{1}^{n+1}-x_{1}^{*}\right\| \\
& \leq\left(1-\alpha^{n}\right)\left\|x_{1}^{n}-x_{1}^{*}\right\|+\alpha^{n}\left[\left(1+\gamma\|A\|^{2} \theta_{2}\right)\left\|y^{n}-x_{1}^{*}\right\|\right]+\alpha_{n} \gamma\|A\| \tau_{2} \varepsilon_{2}^{n}+\alpha^{n}\left\|e^{n}\right\| \\
& \leq\left(1-\alpha^{n}\right)\left\|x_{1}^{n}-x_{1}^{*}\right\|+\alpha^{n} \theta_{1}\left(1+\gamma\|A\|^{2} \theta_{2}\right)\left\|x_{1}^{n}-x_{1}^{*}\right\| \\
& +\alpha^{n}\left[\left(1+\gamma\|A\|^{2} \theta_{2}\right) \tau_{1} \varepsilon_{1}^{n}+\gamma\|A\| \tau_{2} \varepsilon_{2}^{n}+\left\|e^{n}\right\|\right] \\
& =\left[1-\alpha^{n}(1-\theta)\right] \mid x_{1}^{n}-x_{1}^{*} \|+\alpha^{n}\left[\left(1+\gamma\|A\|^{2} \theta_{2}\right) \tau_{1} \varepsilon_{1}^{n}+\gamma\|A\| \tau_{2} \varepsilon_{2}^{n}+\left\|e^{n}\right\|\right]
\end{aligned}
$$

where $\theta=\theta_{1}\left(1+\gamma\|A\|^{2} \theta_{2}\right)$. It follows from the conditions on $\rho_{1}, \rho_{2}$ and $\gamma$ that $\theta \in(0,1)$. Letting

$$
a_{n}=\left\|x_{1}^{n}-x_{1}^{*}\right\|, m_{n}=\alpha^{n}(1-\theta),
$$

$\delta_{n}=\frac{1}{1-\theta}\left[\left(1+\gamma\|A\|^{2} \theta_{2}\right) \tau_{1} \varepsilon_{1}^{n}+\gamma\|A\| \tau_{2} \varepsilon_{2}^{n}+\left\|e^{n}\right\|\right], \forall n \geq 0$. By virtue of (3.13), we have $a_{n+1} \leq\left(1-m_{n}\right) a_{n}+m_{n} \delta_{n}$. Moreover the conditions (i) and (ii) of Lemma 3.1 are satisfied. It follows that $\left\{x_{1}^{n}\right\}$ converges strongly to $x_{1}^{*}$ as $n \rightarrow \infty$.

Since $A$ is continuous, it follows from (25), (26)-(28) that $y^{n} \rightarrow x_{1}^{*}, \quad g_{1}\left(y^{n}\right) \rightarrow g_{1}\left(x_{1}^{*}\right) \quad, \quad A y^{n} \rightarrow A x_{1}^{*}, \quad z^{n} \rightarrow A x_{1}^{*} \quad$ and $g_{2}\left(z^{n}\right) \rightarrow g_{2}\left(A x_{1}^{*}\right)$ as $n \rightarrow \infty$. This completes the proof.

In the following, we consider the convergence of Algorithm 2.3 for SpGMVIP.

Theorem 3.2. For each $i \in\{1,2\}$, let $g_{i}: H_{i} \rightarrow H_{i}$ be $\sigma_{i}$-strongly monotone and $\delta_{i}$-Lipschitz continuous. Let $f_{i}: H_{i} \rightarrow H_{i}$ be $\alpha_{i}$ -strongly monotone with respect to $g_{i}$ and $\beta_{i}$-Lipschitz continuous. Let ${ }_{A: H_{1} \rightarrow H_{2}}$ be a bounded linear operator and $A^{*}$ be its adjoint operator. Suppose $x_{1}^{*} \in \Gamma$ and Assumption 2.2 holds. Then the sequence $\left\{x_{1}^{n}\right\}$ generated by Algorithm 2.3 converges strongly to $x_{1}^{*}$ provided that the constants $\rho_{i}$ and $\gamma$ satisfy the conditions

$$
\begin{gathered}
\mid \rho_{1}-\frac{\alpha_{1}}{\beta_{1}^{2}}<\frac{\sqrt{\alpha_{1}^{2}-\beta_{1}^{2} k_{1}\left(2-k_{1}\right)}}{\beta_{1}^{2}}, \quad \alpha_{1}>\beta_{1} \sqrt{k_{1}\left(2-k_{1}\right)}, \quad k_{1}<1, \\
k_{i}=\sqrt{1-2 \sigma_{i}+\delta_{i}^{2}}, i=1,2, \gamma\|A\|^{2} \theta_{1} \theta_{2}<1-\theta, \quad \gamma \in\left(0, \frac{2}{\|A\|^{2}}\right) \\
\theta_{i}=k_{i}+\sqrt{1-2 \rho_{i} \alpha_{i}+\rho_{i}^{2} \beta_{i}^{2}}, \quad \rho_{i}>0, \mathrm{i}=1,2 .
\end{gathered}
$$

Proof Since $x_{1}^{*} \in \Gamma$, then $x_{1}^{*} \in H_{1}$ is such that $g_{i}\left(x_{i}^{*}\right) \in \operatorname{dom} \varphi_{i}$ and

$$
x_{1}^{*}=x_{1}^{*}-g_{1}\left(x_{1}^{*}\right)+J_{\rho_{1}}^{\partial \varphi_{1}}\left(g_{1}\left(x_{1}^{*}\right)-\rho_{1} f_{1}\left(x_{1}^{*}\right)\right),
$$




$$
A x_{1}^{*}=A x_{1}^{*}-g_{2}\left(A x_{1}^{*}\right)+J_{\rho_{2}}^{\partial \rho_{2}}\left(g_{2}\left(A x_{1}^{*}\right)-\rho_{2} f_{2}\left(A x_{1}^{*}\right)\right),
$$

for $\rho_{i}>0$. From Algorithm 2.3(19), Assumption2.2and (33), we have

$$
\left\|y^{n}-x_{1}^{*}\right\|
$$

$=\left\|x_{1}^{n}-x_{1}^{*}-\left(g_{1}\left(x_{1}^{n}\right)-g_{1}\left(x_{1}^{*}\right)\right)+J_{\rho_{1}}^{\partial \rho^{q}}\left(g_{1}\left(x_{1}^{n}\right)-\rho_{1} f_{1}\left(x_{1}^{n}\right)\right)-J_{\rho_{1}}^{\partial \varphi_{1}}\left(g_{1}\left(x_{1}^{*}\right)-\rho_{1} f_{1}\left(x_{1}^{*}\right)\right)\right\|$

$\leq\left\|x_{1}^{n}-x_{1}^{*}-\left(g_{1}\left(x_{1}^{n}\right)-g_{1}\left(x_{1}^{*}\right)\right)\right\|+\left\|g_{1}\left(x_{1}^{n}\right)-g_{1}\left(x_{1}^{*}\right)-\rho_{1}\left(f_{1}\left(x_{1}^{n}\right)-f_{1}\left(x_{1}^{*}\right)\right)\right\|$

$+\left\|J_{\rho_{1}}^{\partial \rho_{1}^{n}}\left(g_{1}\left(x_{1}^{*}\right)-\rho_{1} f_{1}\left(x_{1}^{*}\right)\right)-J_{\rho_{1}}^{\partial \rho_{1}}\left(g_{1}\left(x_{1}^{*}\right)-\rho_{1} f_{1}\left(x_{1}^{*}\right)\right)\right\|$.

Note that $g_{1}$ is $\sigma_{1}$-strongly monotone and $\delta_{1}$-Lipschitz continuous, and $f_{1}$ is $\alpha_{1}$-strongly monotone with respect to $g_{1}$ and $\beta_{1}$-Lipschitz continuous, we have

$$
\left\|x_{1}^{n}-x_{1}^{*}-\left(g_{1}\left(x_{1}^{n}\right)-g_{1}\left(x_{1}^{*}\right)\right)\right\| \leq k_{1}\left\|x_{1}^{n}-x_{1}^{*}\right\|,
$$

where $k_{1}=\sqrt{1-2 \sigma_{1}+\delta_{1}^{2}}$,

$$
\left\|g_{1}\left(x_{1}^{n}\right)-g_{1}\left(x_{1}^{*}\right)-\rho_{1} f_{1}\left(x_{1}^{n}\right)-{ }_{1} f_{1}\left(x_{1}^{*}\right)\right\| \leq \sqrt{1-2 \rho_{1} \alpha_{1}+\rho_{1}^{2} \beta_{1}^{2}}\left\|x_{1}^{n}-x_{1}^{*}\right\|,
$$

from (33)-(35), we have

$$
\left\|y^{n}-x_{1}^{n}\right\| \leq \theta_{1}\left\|x_{1}^{n}-x_{1}^{*}\right\|+\varepsilon_{1}^{n},
$$

where

$$
\begin{gathered}
\theta_{1}=k_{1}+\sqrt{1-2 \rho_{1} \alpha_{1}+\rho_{1}^{2} \beta_{1}^{2}}, \\
\varepsilon_{1}^{n}=\left\|J_{\rho_{1}}^{\partial \rho_{1}^{p}}\left(g_{1}\left(x_{1}^{*}\right)-\rho_{1} f_{1}\left(x_{1}^{*}\right)\right)-J_{\rho_{1}}^{\partial \rho_{1}}\left(g_{1}\left(x_{1}^{*}\right)-\rho_{1} f_{1}\left(x_{1}^{*}\right)\right)\right\| .
\end{gathered}
$$

Similary ,from Algorithm 2.3(18), Assumption 2.2 and (34),and using the fact that $f_{2}$ is $\alpha_{2}$-strongly monotone with respect to $g_{2}$ and $\beta_{2}$-Lipschitz continuous, $g_{2}$ is

$\sigma_{2}$-strongly monotoneand $\delta_{2}$-Lipschitz continuous, we have

$$
\left\|z^{n}-A x_{1}^{n}\right\| \leq \theta_{2}\left\|A y^{n}-A x_{1}^{n}\right\|+\varepsilon_{2}^{n},
$$

where $\theta_{2}=k_{2}+\sqrt{1-2 \rho_{2} \alpha_{2}+\rho_{2}^{2} \beta_{2}^{2}}, \quad k_{2}=\sqrt{1-2 \sigma_{2}+\delta_{2}^{2}}$,

$$
\varepsilon_{2}^{n}=\left\|J_{\rho_{2}}^{\partial \varphi_{2}^{n}}\left(g_{2}\left(A x_{1}^{*}\right)-\rho_{2} f_{2}\left(A x_{1}^{*}\right)\right)-J_{\rho_{2}}^{\partial \varphi_{2}}\left(g_{2}\left(A x_{1}^{*}\right)-\rho_{2} f_{2}\left(A x_{1}^{*}\right)\right)\right\| .
$$

Combining (29)-(31), (38), (39), we obtain

$$
\begin{aligned}
& \left\|x_{1}^{n+1}-x_{1}^{*}\right\| \\
& \leq\left[1-\alpha^{n}(1-\theta)\right]\left\|x_{1}^{n}-x_{1}^{*}\right\|+\alpha^{n}\left[\left(1+\gamma\|A\|^{2} \theta_{2}\right) \varepsilon_{1}^{n}+\gamma\|A\| \varepsilon_{2}^{n}+\left\|e^{n}\right\|\right],
\end{aligned}
$$

where $\theta=\theta_{1}+\gamma\|A\|^{2} \theta_{1} \theta_{2}$. It follows from the conditions on $\rho_{1}$, $\rho_{2}$ and $\gamma$ that $\theta \in(0,1)$. Letting

$$
\begin{gathered}
a_{n}=\left\|x_{1}^{n}-x_{1}^{*}\right\|, m_{n}=\alpha^{n}(1-\theta), \\
\left.\delta_{n}=\frac{1}{1-\theta}\left[\left(1+\gamma\|A\|^{2} \theta_{2}\right) \varepsilon_{1}^{n}+\gamma\|A\| \varepsilon_{2}^{n}\right)+\left\|e^{n}\right\|\right], \forall n \geq 0 .
\end{gathered}
$$

Then (40) implies

$$
a_{n+1} \leq\left(1-m_{n}\right) a_{n}+m_{n} \delta_{n}, \forall n \geq 0 .
$$

Noting the conditions (i) and (ii) of Lemma 3.1 are satisfied and it follows that $\left\{x_{1}^{n}\right\}$ converges strongly to $x_{1}^{*}$ as $n \rightarrow \infty$. The rest of argument is same as in Theorem 3.3, so is omitted, which is completed the proof.

Remark 3.3. Algorithm 2.2 is a special case of Algorithm 2.1 and Theorem 3.1 extends the corresponding results in [1].

\section{REFERENCES}

[1] K. R. Kazmi, "Split general quasi-variational inequality problem," Georgian Math. J. 22, no.3 , 385-392, 2015.R. Nicole, "Title of paper with only first word capitalized," J. Name Stand. Abbrev., in press.

[2] Y. Censor, A. Gibali and S. Reich, "Algorithms for the split variational inequality problem,” Numer. Algorithms. 59 , no. 2, 301-323, 2012.

[3] Y. Censor, T. Bortfeld, B. Martin and A. Trofimov, "A unified approach for inversion problems in intensity modulated radiation therapy,” Phys. Med. Biol. 51 , no. 10, 2353-2365,2006.

[4] Y. Censor and T. Elfving, "A multiprojection algorithm using Bregman projections in a product space,” Numer. Algorithms. 8 , no. 2-4, 221239, 1994.

[5] P. L. Combettes, "The convex feasibility problem in image recovery," Adv. Imaging Electron. Phys. 95 , 155-270, 1996.

[6] H.K. Xu, "Iterative algorithms for nonlinear operator," Journal of the London Mathematical society. 66, no.1, 240-256, 2002. 